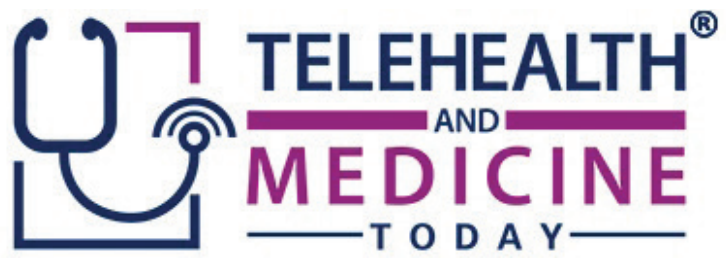

\title{
Interstate Telehealth Policy and Provider Incentives: Recommendations to Combat Historically Low Physician Acceptance of Medicaid Patients
}

\author{
Sophia S. Albanese, Emily A. Yin, Sarah J. Timmons
}

Affiliations: Wellesley College, Wellesley, MA, USA

Corresponding Author: Sophia S. Albanese, sophiaalbanese1@gmail.com.

Keywords: Cross-State Medical Practice, Medicaid, Parity, Reimbursement, Telehealth, Telemedicine, COVID-19

Section: Original Market Research

Methods: A holistic, mixed-methods approach was used to investigate the research goal. Primary source telehealth reimbursement data obtained from the Medicaid offices of Florida and Nebraska, extensive literature review on Medicaid and telehealth policy, as well as secondary source data from online databases and previously published research were used to highlight the improvements needed to better implement telehealth programs across the country, as well as to identify precedential cases of policy changes regarding telemedicine.

Results: The primary and secondary source data analyzed in this article demonstrate the need for policy changes to address historically low physician acceptance of new patients through state Medicaid. Data obtained from Florida and Nebraska in Table 1 corroborate with the data in Table 2-both demonstrating how Florida reimburses at a much lower rate
(0.79 to national Medicaid average) as compared to Nebraska (1.14 to national Medicaid average). Table 3 exhibits how national averages for Medicaid reimbursement, as well as Florida's and Nebraska's averages, fall below the national averages for Medicare reimbursement in all categories except for obstetric care, showing that Medicaid services are reimbursed at a lower rate than Medicare in most circumstances.

Conclusions: Nationally, Medicaid reimbursement rates are among the lowest reimbursement rates of any insurer. Additionally, new Medicaid patients witness the lowest rates of acceptance by physicians, in large part due to low reimbursement rates. Medicaid policies and reimbursement rates vary across each state, making it difficult to enact any broadsweeping policies to improve the access to care for Medicaid beneficiaries in the United States. However, by drawing reference to several policy changes involving Medicare, Medicaid, and 
telehealth, this article presents recommendations for an incentivized cross-state telehealth policy aimed at increasing Medicaid beneficiaries' access to care. With nation-wide policy changes like those during the COVID-19 pandemic, there are historical examples and precedence to support policies focused on decreasing the limitations and barriers needed to practice telemedicine across state lines. This article offers a potential, but limited framework for states to consider implementing in their Medicaid programs after conducting further research on the state-by-state level.

\section{7 he Department of Health and Human Services defines telehealth as 'the use of electronic information and} telecommunication technologies to extend care when you and the doctor aren't in the same place at the same time. ${ }^{1}$ In general, telemedicine can range across a variety of forms, whether it is through an app on a patient's phone, a virtual appointment with a doctor via live video, or even a virtual consult from a distant physician while at a hospital; however, the reimbursement rates for these services may differ by insurance provider, or the services may not be offered at all. ${ }^{2}$ Despite these varying policies, telemedicine is widely known for its ability to increase access to care for rural or underserved patient populations. $^{3}$

This article analyzes a common issue within state Medicaid fee-for-service: the connection between low reimbursement rates and low physician acceptance of new patients. The article presents data and state policies used to advocate for a potential modification of telehealth regulations for the Medicaid fee-for-service population in an effort to increase access to care for new Medicaid patients initially denied by their providers. Previous examples of changes in telehealth policy under Medicare and Medicaid, such as policy changes related to the COVID-19 pandemic, are used to represent historical precedence for the recommendations provided within the article.

\section{METHODS}

A mixed-methods approach involving analysis of raw data and utilization of literature review, online resources, previously published research, and databases was used to investigate the research problem. This study examines telemedicine reimbursement data specifically under fee-forservice Medicaid rather than managed care plans, due to the fact that managed care reimbursement rates can vary from plan to plan within each individual state. Fee-for-service avoids this variability by having one reimbursement rate per code in each state Medicaid plan. ${ }^{4}$ Using feefor-service in this study allowed for results to be more easily compared across state Medicaid plans, as well as allowing for future research to be conducted more seamlessly in order to investigate potential scenarios in other locations across the United States.

In an effort to gain more insights into the current telehealth landscape within Medicaid fee-for-service telemedicine, our team solicited telemedicine reimbursement information from Medicaid offices, departments of health, offices of health and human services, regional telehealth resource centers, and various other state offices and organizations across all 50 states and the District of Columbia via email and phone under the Freedom of Information Act. Among the states that responded with data, the provided information was analyzed and crosschecked for common data points, which would allow the research team to identify cross-state comparisons.

Two states, Florida and Nebraska, were chosen to analyze for the purpose of this study based on 
the information these states provided. Florida was chosen to represent the non-parity Medicaid states within the United States. Florida's Medicaid policies have no mention of payment parity, which means that there is no requirement for telehealth services to be reimbursed at the same rate as in-person services. ${ }^{5}$ Nebraska was chosen to represent states that reimburse not only with parity but also reimburse at higher rates than national Medicaid averages. Nebraska Medicaid reimburses at $114 \%$ of the national average of all state Medicaid offices, while Florida Medicaid reimburses at a rate of $79 \%$ of the national average. ${ }^{6}$ The policies proposed in this study are simply a recommendation to be considered by Medicaid offices, and is not currently functional between the states of Florida and Nebraska at the time this article was written.

Florida and Nebraska Medicaid provided the Current Procedures Terminology (CPT) code and descriptions for the codes reimbursed by Medicaid via telehealth in their state, along with the reimbursement rates for each service within the given year. The data provided by Florida did not distinguish reimbursement rates between types of provider, whereas Nebraska recorded rates for various provider types such as $\mathrm{MD}$ / DO, PA, and Dental. For the purpose of this study, Nebraska MD rates were used to compare with Florida's provider rates. In the cases where an MD rate was not available for a Nebraska code, the physician rate was used. To determine the codes to analyze for the study, the Florida and Nebraska codes were cross-referenced in order to identify the in-common codes between the two states within the 2017-2018 year. This resulted in nine mutual fee-for-service codes and reimbursement rates, as shown in Table 1, in which the percent differences in average reimbursement rates were calculated in order to allow for comparison and further analysis of the common data between the two states.
In addition to the data provided by Florida and Nebraska Medicaid, a literature review was conducted on the current and historical policy landscape of telehealth through Medicaid and Medicare. Focus was given to literature in reputable medical, health, telehealth, and policy-related online journals and publications published within the last 15 years. Topic areas for review included state Medicaid regulations regarding telemedicine, Interstate Medical Licensure, policy changes to increase the use of telemedicine, and the impact of telemedicine on access to care within the United States across different insurance providers.

Along with literature review, online databases and websites, such as the Center for Connected Health Policy and Nebraska and Florida Medicaid websites, were utilized to obtain the most up-to-date state-by-state policies and regulations on telemedicine, Medicaid reimbursement, historical telehealth policy changes, and the overall landscape of healthcare across states. This information was used not only as a way to best construct the current understanding and functionality of telemedicine today, but also to highlight commonly referenced areas for improvement within the way states practice and reimburse for services provided via telemedicine and how these improvements might be applied in tangible cross-state telemedicine policies like the ones proposed in this article.

The use of several methods allowed for a holistic approach that, with further research and increased telemedicine usage data from each state, could lead to an incentivized, cross-state framework for telemedicine via Medicaid fee-for-service that would aim to eliminate the historically low physician acceptance of new Medicaid patients across the United States. 


\section{RESULTS}

Using a mixed-methods approach, results for the investigation include primary source data sets provided by the Florida and Nebraska Medicaid offices, as well as other relevant secondary source data and literature focusing on the reimbursement landscape of Medicaid. All of these results are available to the public in online publications or via the Freedom of Information Act.

Information received from Florida's Medicaid Office via the Freedom of Information Act comprised discreet data collected from both telemedicine and non-telemedicine encounters for 2016-2017 and 2017-2018 under Medicaid fee-for-service. The data included the number of distinct recipients, claim lines, and the reimbursement amounts per procedure code (CPT) under fee-for-service as well as Managed Care Medicaid within Florida. Information was categorized by year, with 16 telemedicine feefor-service CPT codes reported in 2016-2017 and 20 in 2017-2018.

Information from Nebraska's Medicaid Office provided a list of CPT and Healthcare Common Procedure Coding System (HCPCS) codes covered when performed via telehealth using the GT modifier (for telehealth claims, modifier means "via interactive audio and video telecommunications systems) for 2018-2019." Along with the codes was the description of each service and the rate at which each service is reimbursed when performed by various practitioners, including MD, DO, PA, APRN, Dental, and Vision.

The data set received from Nebraska is congruent with the Medicaid Fee-for-Service Physician Fee Schedule found on the website of Nebraska Medicaid Department of Health and Human Services. ${ }^{7}$ Using the Nebraska Medicaid website, it became clear that the rates for the codes identified in Table 1 were reimbursed at the same rates in 2017-2018 and 2018-2019. For this reason, we were able to compare the 2017-2018 reimbursement rates from Florida and Nebraska, as shown in Table 1.

Values from Florida were calculated as the total amount reimbursed by Medicaid per procedure code divided by the number of claims within a given year for that code, resulting in the average reimbursement rate per code. Nebraska's values are not linked to a population; rather, they are the statemandated reimbursement rates for each particular code, which do not vary unless changed by the state. Nebraska's telehealth rates are reimbursed at an equal rate to the equivalent in-person services due to payment parity within the state. ${ }^{8}$ The "\% difference" was calculated using the formula (Nebraska Rate - Florida Rate) / ((Nebraska Rate + Florida Rate) $/ 2)) \times 100$, rounded to the second decimal using Excel functions.

Table 2 exhibits data collected by Zuckerman et al. ${ }^{9}$ from the Urban Institute's report on Medicaid physician fees. The data show how Florida's and Nebraska's Medicaid fee schedules compare to the national average of all state Medicaid reimbursement rates. Florida Medicaid is measured below the national average for fee-for-service reimbursement in all categories except for obstetric care, where it is $5 \%$ above the national reimbursement average. Nebraska Medicaid reimburses above the national average for fee-for-service in all the categories analyzed. Of all 50 US states and the District of Columbia, 20 states fall below the national reimbursement average for Medicaid reimbursement, while 30 rank above the national average. Tennessee is not included here, as a fee-for-service program does not exist in the state. ${ }^{6}$

As this article investigates and as history demonstrates, Medicaid services are reimbursed 
Table 1. Comparing reimbursement rates for nine telemedicine codes in Florida and Nebraska Medicaid Fee-for-Service Reimbursement Rates for Telemedicine Services, 2017-2018

\begin{tabular}{|lccc|}
\hline Procedure code & Florida Medicaid $(\$)$ & Nebraska Medicaid(\$) & \% difference \\
\hline 90791 & 76.18 & 179.32 & 80.74 \\
\hline 90792 & 64.79 & 250.77 & 117.87 \\
\hline 99211 & 2.13 & 32.18 & 175.17 \\
\hline 99213 & 29.02 & 64.45 & 75.81 \\
\hline 99214 & 50.33 & 88.80 & 55.3 \\
\hline 99252 & 43.19 & 65.68 & 41.32 \\
\hline 99253 & 66.14 & 94.30 & 35.1 \\
\hline 99254 & 95.00 & 110.70 & 15.26 \\
\hline 99255 & 114.33 & 126.28 & 9.93 \\
\hline
\end{tabular}

Source: Office of Public Records at the Agency for Health Care Administration for the State of Florida; Public Records Department for the Health and Human Services Department for the State of Nebraska.

Table 2. Comparing state and national reimbursement rates under Medicaid fee-for-service. ${ }^{9}$

\begin{tabular}{|lccc|}
\hline Medicaid Physician Fee Index, 2016 & United States & Florida & Nebraska \\
\hline All services & 1 & 0.79 & 1.14 \\
\hline Primary care & 1 & 0.74 & 1.02 \\
\hline Obstetric care & 1 & 1.05 & 1.14 \\
\hline Other services & 1 & 0.75 & 1.45 \\
\hline
\end{tabular}

Table 3. Comparing Medicaid fee-for-service reimbursement rates with Medicare fee-for-service reimbursement rates. ${ }^{9}$

\begin{tabular}{|lccc|}
\hline Medicaid-to-Medicare Fee Index, 2016 & United States & Florida & Nebraska \\
\hline All services & 0.72 & 0.56 & 0.92 \\
\hline Primary care & 0.66 & 0.48 & 0.71 \\
\hline Primary care for physicians eligible for increased fee & null & 0.53 & 1.01 \\
\hline Obstetric care & 0.81 & 0.82 & 1.05 \\
\hline Other services & 0.82 & 0.58 & 1.33 \\
\hline
\end{tabular}

at lower rates than Medicare and private payers for the same services. ${ }^{10}$ Table 3 , consisted of information collected from the Urban Institute's study, demonstrates the phenomenon that Medicaid reimburses at lower rates across the country than Medicare for all fee-for-service codes. ${ }^{9}$

As shown in Table 3, the average of "All Services" under Medicaid within the
United States are reimbursed at $72 \%$ of the Medicare national average. Florida Medicaid reimburses at $56 \%$ of Florida Medicare rates for "All Services," while Nebraska reimburses at $92 \%$ of the Medicare reimbursement average for "All Services" in Nebraska. Florida is below the national reimbursement average, while Nebraska is above the national reimbursement average of Medicaid-to-Medicare Reimbursement Fees. 


\section{DISCUSSION}

The data presented above show that physicians are reimbursed at a lower rate when treating Medicaid patients than for Medicare patients for the same services. The data also highlight how Florida Medicaid reimburses at a lower rate ( $79 \%$ of the national average) compared to Nebraska Medicaid (114\% of the national average). ${ }^{9}$

The results of studies reveal that low reimbursement rates impact providers' likelihood to treat patients. Research conducted by the Medicaid and Children's Health Insurance Program (CHIP) Payment and Access Commission (MACPAC) in 2019 showed that providers are $70.8 \%$ likely to accept a new Medicaid-insured patient, $85.3 \%$ likely to accept a new Medicare-insured patient, and $90.0 \%$ likely to accept a new privately insured patient. ${ }^{11}$ These statistics show disparity in physician access for the Medicaid population compared to other insurers. The MACPAC study also found that physicians located in states with a high Medicaid-to-Medicare reimbursement ratio, such as Nebraska, are $81.1 \%$ likely to accept a new Medicaid-insured patient, whereas physicians in states with low Medicaid-to-Medicare reimbursement ratios, such as Florida, are only $64.5 \%$ likely to accept and treat new Medicaid-insured patients. ${ }^{11}$ Based on this information about physician preference as well as the data presented in Table 3 about Medicaid-to-Medicare reimbursement rates, connections can be drawn between reimbursement rates for Medicaid services and the likelihood of physicians to accept new Medicaid patients. As found by the Medicaid and CHIP Payment and Access research, physicians are shown to be more likely to accept and treat Medicaid patients in states where the fee-for-service reimbursement rates are more closely reimbursed to the Medicare reimbursement rates within that state. ${ }^{11}$

Table 2 shows that Florida Medicaid reimburses providers at $79 \%$ of the national Medicaid reimbursement average in the United States. ${ }^{9}$ Studies point to low reimbursement rates compared to other insurers as one of the main reasons physicians deny treating new Medicaid patients. ${ }^{12}$ According to the General Medicaid Policy for the state of Florida, physicians reserve the right to refuse service for any reason, except for emergencies, patient inability to pay a copay at the time of service, or for any discriminatory reasons against race, beliefs, or disability of the patient. ${ }^{13}$ This allows Florida physicians to deny acceptance of a new Medicaid patient for any other reason, which includes denial due to low reimbursement rates for their plan. Recent healthcare policies like the Affordable Care Act and increased implementation of Managed Care plans have aimed to augment Medicaid coverage by increasing reimbursement rates and extending coverage to more Americans, but recent research has proved that these policies did not show significant increase in physician acceptance of new Medicaid patients; however, the policies successfully provided insurance coverage to a larger population. ${ }^{12,14}$

Surveys by the National Center for Health Statistics show that $40.9 \%$ of Florida physicians and $13.0 \%$ of Nebraska physicians did not accept new Medicaid patients in 2011. ${ }^{15}$ Following the logic of aforementioned studies, lower reimbursement rates for services coincide with higher denial rates of new Medicaid patients. That said, Florida's relatively low reimbursement rates would correlate with a 
higher denial rate of new Medicaid patients, while Nebraska's relatively high reimbursement rates would correlate with a lower denial rate. The data received from the Medicaid offices of Florida and Nebraska (see Table 1) corroborate this theory and aid in explaining why Florida physicians are $27 \%$ less likely to accept new Medicaid patients as compared to Nebraskan physicians. ${ }^{15}$ While there appears to be a correlation between reimbursement rates and acceptance rates of new Medicaid patients, further research is needed to determine the causes.

Of the codes surveyed, Florida Medicaid reimburses at a lower rate than Nebraska Medicaid for all nine codes. Code 99211, an office or outpatient visit/evaluation of an established patient, shows a $1,410.8 \%$ difference between Florida's and Nebraska's reimbursement rates according to the 20172018 data provided by the states. This scenario emphasizes how drastic the disparity of Medicaid reimbursement rates can be across states. Moreover, as shown in Table 3, Florida Medicaid reimburses at about half the rate of Medicare, making practicing on Medicare and privately insured patients more profitable for physicians than practicing on Medicaid patients. ${ }^{16}$

Based on this cost information and the findings of several studies on lack of physician acceptance of Medicaid patients, Medicaid patients are shown to run the risk of limited access to care compared to Medicare or privately insured patients. This study recommends a potential framework to utilize telehealth technologies and state partnerships in an effort to decrease the amount of Medicaid patients across the country who are denied access to care compared to beneficiaries of other insurance providers.
The policy landscape surrounding telehealth has changed widely over the past decade. In most of these cases, a telehealth policy is designed to resolve a certain issue affecting a patient population. The following policies and regulations were taken into consideration when drafting the overarching policy recommendation.

Precedent 1-COVID-19: One of the most recent changes to telehealth policy relates to the COVID-19 pandemic, where the Center for Medicare and Medicaid Services (CMS) temporarily modified several key policies regarding the utilization and practice of telehealth services on the state and national levels in an effort to curtail the spread of the virus by minimizing the amount of inperson contact and allowing for more virtual consultations via telemedicine. ${ }^{17}$ According to CMS, a temporary addition of 85 new reimbursable telemedicine codes are now reimbursable under Medicare due to the Public Health Emergency declared over the coronavirus. ${ }^{18}$ These codes cover many services, including those for "new patients" and services where the patient is located at their own home. Additionally, the temporary policy eliminates the requirement for a patient to be located in a rural area and waives licensure laws for providers. ${ }^{4}$ Prior to these temporary changes, Medicare patients could only utilize telemedicine services if they were located in a designated rural area, if they had an established relationship with their physician, and if the provider has an individual license for each state in which they intend to practice telemedicine. ${ }^{17}$ Medicaid policies related to COVID-19 vary by state; however, all 50 states and the District of Columbia have released updates related to telehealth usage during the pandemic. ${ }^{19}$ These policy modifications, however temporary, represent unprecedented access to 
telemedicine in the Medicare and Medicaid patient populations.

\section{Precedent 2-Bipartisan Budget Act: An} additional example of telehealth policy being transformed in order to improve access to care for a certain population involves the Bipartisan Budget Act of 2018. In this act, changes were made to the originating site requirements for Medicare beneficiaries with end-stage renal disease and acute stroke, allowing the home and mobile stroke units to be eligible originating sites for telemedicine. ${ }^{20,21}$ Unlike the COVID-19 policy changes, the Bipartisan Budget Act is not temporary, yet both aim to provide increased access to care for a certain population who, without these added policies, may be unable to receive appropriate medical attention at the same cost.

\section{Precedent 3-Medicaid Electronic Health} Record Incentive Program: In 2011, the Center for Medicare and Medicaid Services established a plan in connection with the Affordable Care Act that would provide incentives for Medicaid providers and hospitals who adopt and continually utilize electronic health records. ${ }^{22}$ Certain eligibility requirements, such as the percentage of Medicaid patients treated by the provider, must be achieved by practitioners in order to receive the monetary incentive, which could be as much as $\$ 63,750$ over the course of 6 years. ${ }^{23}$ This program provides a key example of Medicaid's focus on the modernization of coverage for its beneficiaries, ${ }^{24}$ and the form of a monetary incentive program sets a precedent for future incentivized programming through Medicaid.

Precedent 4-SUPPORT Act: In an attempt to increase coverage and treatment for patients with substance-use abuse, CMS instituted the Support Use-Disorder Prevention that Promotes
Opioid Recovery and Treatment for Patients and Communities Act in 2019. This policy allows telehealth services related to substance abuse and co-occurring mental health disorders to be performed at the patient's home. ${ }^{25,26}$ This modification of the Medicare policy exemplifies how the use of telemedicine can be used to increase access to care for certain critical patient populations by eliminating barriers such as low reimbursement or restrictive regulations. ${ }^{27}$

\section{Precedent 5-Interstate Medical Licensure} Compact (IMLC): In addition to the governmental policies addressed above, the IMLC was drafted in 2014, with its first operational model being released in 2017, to address a rising issue around the costly and timely process involved in obtaining state medical licensure. ${ }^{28}$ As a result of the increased use of telemedicine, the need for state-by-state licensure has been cited as one of the major barriers to implementation of telehealth, as it limits providers to practice only within the states in which they have a license. ${ }^{29}$ For this reason, the IMLC can be utilized as a way to not only facilitate simpler and more cost-effective ways for practitioners to obtain various licenses, but also as a way to catalyze cross-state telemedicine practice. ${ }^{30}$ The Compact allows physicians from member states to practice in other member states through the agreement to honor each state's medical licensure laws. ${ }^{31}$ Currently 29 states are members of the Interstate Medical Licensure Compact. $^{28}$

The five aforementioned policies and programs exemplify the historical precedence within Medicare and Medicaid to alter current policy in order to increase access to care via telehealth, as well as the shift toward cross-state practice exemplified by the increased utilization of the IMLC. In each scenario, a particular 
issue related to a subset of beneficiaries was targeted, the most prevalent being the need for increased access to care for patients with certain health conditions. The changes made in these policies were taken into consideration when developing this manuscript's potential solution to the historical lack of care for new Medicaid patients, as was analyzed in the data in Tables 1-3.

\section{RECOMMENDATIONS}

The policy proposed in this article encompasses several of the precedential cases explored above in an effort to combat the historically low acceptance rates of Medicaid patients across the country as examined earlier in this article. For the first aspect of the policy, state agencies should consider modeling or encouraging programs similar to Precedent 5, the IMLC. Cross-state telemedicine serves a major role in the increased access to care for patients who otherwise struggle to receive care, whether it is due to a geographic restriction or due to a lack of medical personnel in the area. ${ }^{32}$ However, individual state-by-state licensure requirements introduce financial and regulatory burdens on providers applying for cross-state or multiple-state licensure, potentially deterring providers from obtaining multiple licenses and therefore limiting their ability to practice telemedicine outside of their state. ${ }^{33}$ Given the ability of telehealth to enhance quality of care while surpassing boundaries, state licensure laws impede the number of physicians who wish to practice out of state using telemedicine. With physicians able to practice across state lines using virtual technology, this policy could allow increased access to care for the Medicaid population, extending not only across their state, but also across the country. Considering the expected physician shortage in the United States within the next decade, ${ }^{34}$ cross-state telemedicine practice could play a crucial role in the Medicaid population and beyond.

In order for providers to practice on patients via telemedicine, state Medicaid and Medicare offices hold certain requirements regarding the need for an established patient-provider relationship prior to a telemedicine interaction, as well as the location of the patient during the interaction. ${ }^{4,20,25,35}$ Policy changes during the COVID-19 pandemic, the Bipartisan Budget Act, and SUPPORT Act lessened these requirements in order to allow for increased access to care for specific patient populations who may have difficulty receiving traditional care. State Medicaid offices should consider eliminating the requirements of preexisting patient-provider relationships for telehealth encounters for Medicaid patients who have been denied access to care by an in-person physician. Additional measures, such as broadening eligible originating sites, should be considered if issues of high denial rates for new Medicaid patients persist. State Medicaid offices should consider implementing policies like these, specifically for the population of new Medicaid recipients who are initially denied in-person access to care by a provider.

Based on the changes made in the Affordable Care Act regarding incentivized programs, state Medicaid offices could be encouraged to accept new Medicaid patients in their practice in return for a monetary incentive. As the Medicaid Electronic Health Record Incentive Program requires providers to treat a minimum volume of $30 \%$ Medicaid patients in order to receive the incentive, ${ }^{23}$ providers should only be eligible for an incentive if they accept the designated percentage of new Medicaid patients on a yearly basis. This percentage should be decided and agreed upon by the states. 
These recommendations are summarized below in a flow chart showing the path in which policies can be enacted and utilized to address the historical issues related to limited access to care for new Medicaid patients (see Recommendations 1 and 2).

\section{CONCLUSIONS}

This study proposes a potential policy framework to be utilized by state Medicaid offices in the attempt to increase access to care within the Medicaid population. Connections are drawn between data observed on low reimbursed rates for Medicaid and the high rates of physician

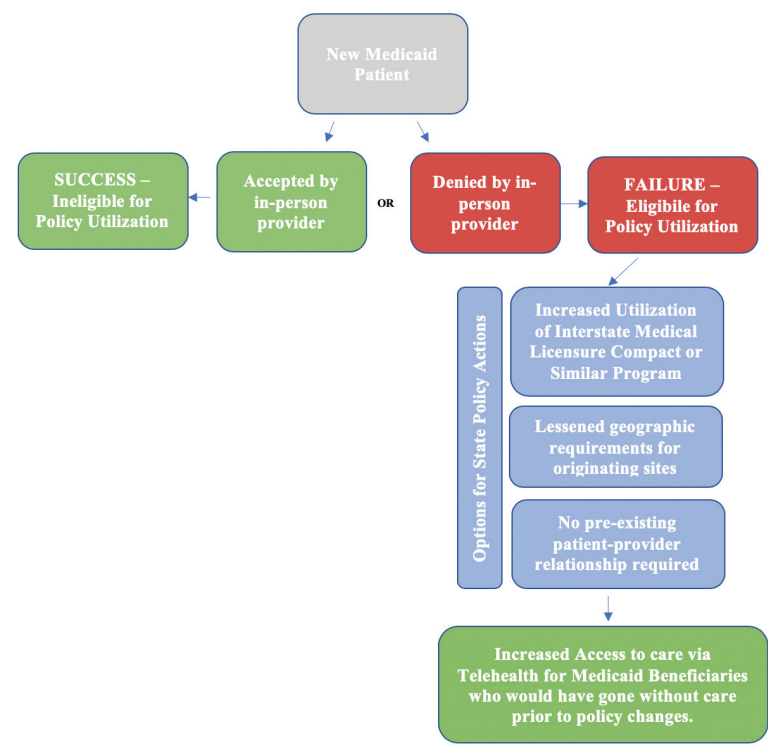

Recommendation 1-Policy-based approach for increased telemedicine utilization. denial for new Medicaid patients. The data demonstrate the need for state and federal agencies to give more attention to this subset of the Medicaid population and enact policies that will increase access to care. The policy recommendations made in this article are drawn upon precedential changes in healthcare policy, particularly with regard to the utilization of telehealth as a way to surpass boundaries in access to care for certain vulnerable populations.

The recommendations, although not allencompassing, propose the implementation of policy changes to allow for increased cross-state telemedicine practice, modifications to eligible originating sites and pre-existing patient-provider relationships via telemedicine, or incentivizing the in-person practice on new Medicaid patients. If enacted, these policies would largely benefit the Medicaid populations in states with low Medicaidto-Medicare reimbursement rates, such as Florida, where providers denied $40.9 \%$ of new Medicaid patients in $2011^{15}$ - a much higher denial rate than in states where Medicaid reimbursement is higher.

Studies anticipate rising enrollment into Medicaid programs across the country as a result of the economic downturn associated with the COVID19 pandemic. ${ }^{36,37}$ As the data in Table $1-3$ demonstrate, current Medicaid beneficiaries already experience difficulties accessing care from physicians who deny new Medicaid patients.

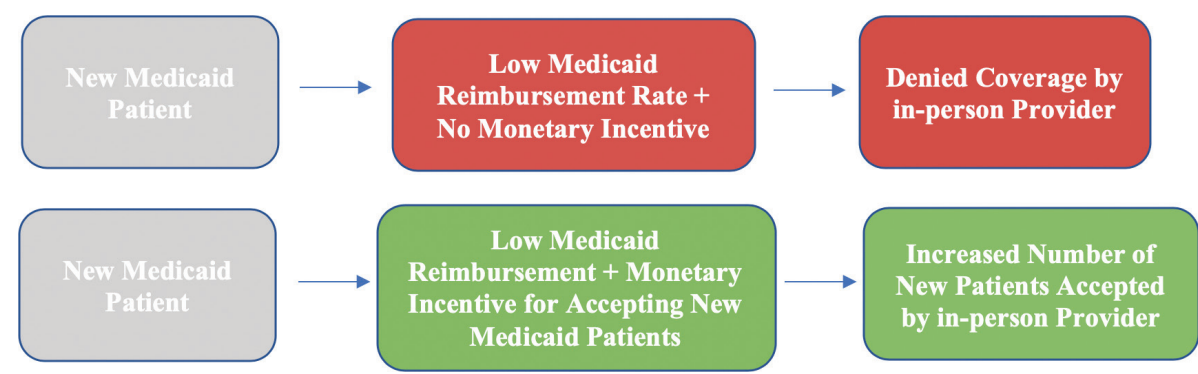

Recommendation 2-Incentivized approach for increased telemedicine utilization. (Applies mainly to states where the Medicaid reimbursement rate is lower than the rates for other insured groups). 
With the expected increase in enrollees, this issue should be addressed in order to proactively advocate for Medicaid patients needing care.

The policies recommended in this article do not directly address issues raised about low reimbursement rates for Medicaid compared to other plans. Further research should be conducted at the state and national levels to determine the policies that could best mitigate this, and other issues not analyzed in this article.

\section{Conflict of Interests}

The authors declare no potential conflicts of interest.

\section{Contributors}

All authors contributed to the manuscript creation and to the project.

\section{Funding Statement}

None.

\section{REFERENCES}

1. Telehealth.HHS.gov. Understanding Telehealth. Available from: https:// telehealth.hhs.gov/patients/understandingtelehealth/

2. Center for Connected Health Policy. State Telehealth laws \& reimbursement policies. Available from: https://www.cchpca. org/sites/default/files/2019-10/50\%20 State\%20Telehalth\%20Laws\%20and\%20 Reibmursement\%20Policies\%20Report\%20 Fall\%202019\%20FINAL.pdf

3. Muzammil, Sadat. Telehealth: Is it only for the rural areas? A review of its wider use. TMT. 2020;5(1):1-16. https://doi. org/10.30953/tmt.v5.162

4. Center for Connected Health Policy. COVID-19 Telehealth coverage policies. Available from: https://www.cchpca.org/ resources/covid-19-telehealth-coveragepolicies
5. Center for Connected Health Policy. Current state laws \& reimbursement policies. Available from: https://www. cchpca.org/telehealth-policy/current-statelaws-and-reimbursement-policies?jurisdicti on $=38 \&$ category $=$ All\&topic $=3$

6. Kaiser Family Foundation. Medicaid physician fee index. Available from: https:// www.kff.org/medicaid/state-indicator/ medicaid-fee-index/?currentTimeframe $=0 \&$ sortModel $=\% 7 \mathrm{~B} \% 22$ colId $\% 22: \% 22$ Locatio $\mathrm{n} \% 22, \% 22$ sort $\% 22: \% 22$ asc $\% 22 \% 7 \mathrm{D}$

7. Nebraska Department of Health and Human Services. Medicaid provider rates and fee schedules. Available from: http://dhhs. ne.gov/Pages/Medicaid-Provider-Ratesand-Fee-Schedules.aspx?Paged =TRUE\&p Fee_x0020_Schedule $=$ Injectables\&p Effective_x0020_Date $=20190101 \% 20$ 06\%3A00\%3A00\&p_ID=131\&PageFirst Row $=91 \& \& V i e w=\% 7 B 231 D B 632-0133-$ 4683-B02E-FE97BDE343E5\%7D

8. State Plan Under Title XIX of the Social Security Act-Methods and Standards for Establishing Payment Rates 2019 (DHHS NE). Available from: https:// files.nc.gov/ncdma/NC-State-Plan-ForMedical-Assistance-Programs-2019-11-05-bjs.pdf

9. Zuckerman S, Skopec L, Epstein M. Medicaid physician fees after the ACA primary care fee bump. Urban Institute. 2017:1-13. Available from: https://www. urban.org/research/publication/medicaidphysician-fees-after-aca-primary-care-feebump/view/full_report

10. Nasseh K, Vujicic M, Yarbrough C. A ten-year, state-by-state, analysis of Medicaid fee-for-service reimbursement rates for dental care services. Health Policy Institute Research Brief. American Dental Association. 2014. Available from: http://www.ada.org/ /media/ADA/ Science\%20and\%20Research/HPI/Files/ HPIBrief_1014_3.ashx

11. Holgash, K., \& Heberlein, M. Physician acceptance of new Medicaid patients: What matters and what doesn't. Available from: 
https://www.healthaffairs.org/do/10.1377/ hblog20190401.678690/full/

12. Holgash K, Heberlein M. Physician acceptance of new Medicaid patients: What matters and what doesn't. https://doi. org/10.1377/hblog20190401.678690

13. Agency for Health Care Administration. Florida Medicaid: General Medicaid policy. Available from: https://ahca. myflorida.com/medicaid/review/Rules_ in_Process/Develop/59G-1.050_General_ Medicaid_Policy.pdf

14. Mazurenko O, Casey CP, Agarwal R, Carroll RA, Menachemi N. The effects of Medicaid expansion under the ACA: A systematic review. Health Affairs. 2018;37(6). https://doi.org/10.1377/ hlthaff.2017.1491

15. Decker S. In 2011 nearly one-third of physicians said they would not accept new Medicaid patients, but rising fees may help. Health Affairs. 2012;31(8):1673-9. https:// doi.org/10.1377/hlthaff.2012.0294

16. Kaiser Family Foundation. Medicaidto-Medicare fee index. Available from: https://www.kff.org/medicaid/stateindicator/medicaid-to-medicare-fee-index/ ?currentTimeframe $=0 \&$ sortModel $=\% 7 \mathrm{~B} \%$ 22 colId $\% 22: \% 22$ Location $\% 22, \% 22$ sort $\% 2$ 2:\% $\% 22$ asc $\% 22 \% 7 \mathrm{D}$

17. Centers for Medicare \& Medicaid Services. Medicare telemedicine health care provider fact sheet. Available from: https://www. cms.gov/newsroom/fact-sheets/medicaretelemedicine-health-care-provider-fact-sheet

18. Center for Medicare and Medicaid Services. List of Telehealth services. Available from: https://www.cms.gov/Medicare/MedicareGeneral-Information/Telehealth/TelehealthCodes

19. Center for Connected Health Policy. COVID-19 related state actions. Available from: https://www.cchpca.org/resources/ covid-19-related-state-actions

20. Center for Connected Health Policy. National policy. Available from: https:// www.cchpca.org/telehealth-policy/ telehealth-and-medicare
21. Center for Medicare and Medicaid Services. Final policy, payment, and quality provisions changes to the Medicare physician fee schedule for calendar year 2019. Available from: https://www.cms.gov/ newsroom/fact-sheets/final-policy-paymentand-quality-provisions-changes-medicarephysician-fee-schedule-calendar-year

22. Center for Medicare and Medicaid Services. CMS finalizes requirements for the Medicaid Electronic Health Records (EHR) incentive program. Available from: https:// www.cms.gov/newsroom/fact-sheets/cmsfinalizes-requirements-medicaid-electronichealth-records-ehr-incentive-program

23. Center for Medicare and Medicaid Services. Medicaid state information. Available from: https://www.cms. gov/Regulations-and-Guidance/ Legislation/EHRIncentivePrograms/ MedicaidStateInfo

24. Medicaid.gov. Encouraging investment in Medicaid information technology. Available from: https://www.medicaid.gov/about-us/ messages/entry/47649

25. Center for Medicare and Medicaid Services. Telehealth Services. Available from: https://www.cms.gov/Outreachand-Education/Medicare-LearningNetwork-MLN/MLNProducts/ downloads/TelehealthSrvcsfctsht. pdf?utm_campaign=2a178f351b-EMAIL CAMPAIGN_2019_04_19_08_59\&utm term $=0$ ae $00 \bar{b} 0 \mathrm{e} 89 \mathrm{a}-2 \mathrm{a} 178 \mathrm{f} 351 \mathrm{~b}-$ $353229765 \& u t m \_$content $=90024811 \& u t m$ medium $=$ social\&utm_source $=$ linkedin\&hss_channel=lcp-3619444

26. H.R.6-Substance use-disorder prevention that promotes opioid recovery and treatment for patients and communities act or the SUPPORT for patients and communities act 2018. https://nhchc.org/wp-content/ uploads/2019/08/national-hch-council-_-hr6-legislation-synopsis.pdf

27. Huskamp HA, Busch AB, Souza J, et al. How is telemedicine being used in opioid and other substance use disorder treatment? Health Aff (Millwood). 
2018;Dec; 37(12):1940-1947. https://doi. org/10.1377/hlthaff.2018.05134

28. Interstate Medical Licensure Compact. A faster pathway to physician licensure. Available from: https://www.imlcc.org/afaster-pathway-to-physician-licensure/

29. Jacobson PD, Selvin E. Licensing telemedicine: The need for a national system. Telemedicine and e-Health. 2004;6(4):424-39. https://doi. org/10.1089/15305620050503915

30. Steinbrook R. Interstate medical licensure: Major reform of licensing to encourage medical practice in multiple states. JAMA. 2014;312(7):695-96. https://doi. org/10.1001/jama.2014.9809

31. Chandrashekar P, Jain SH. Eliminating barriers to virtual care: implementing portable medical licensure. Am J Managed Care. 2019;26(1):20-2. https://doi. org/10.37765/ajmc.2020.41223

32. Ashwood JS, Mehrotra A, Cowling D, Uscher-Pines L. Direct-to-consumer telehealth may increase access to care but does not decrease spending. Health Affairs. 2017;36(3):485-91. https://doi.org/10.1377/ hlthaff.2016.1130

33. Becker CD, Dandy K, Gaujean M, et al. Legal perspectives on telemedicine part 1: Legal and regulatory issues. Perm J. 2019;23:18-293. https://doi.org/10.7812/ TPP/18-293

34. Zhang X, Lin D, Pforsich H, Lin VW. Physician workforce in the United States of
America: Forecasting nationwide shortages. Hum Resour Health. 2020;18(8). https://doi. org/10.1186/s12960-020-0448-3

35. American Medical Association. 50state survey: Establishment of a patient-physician relationship via telemedicine. Available from: https:// www.ama-assn.org/system/files/2018-10/ ama-chart-telemedicine-patient-physicianrelationship.pdf

36. Weinstein RS, et al. Telemedicine, telehealth, and mobile health applications that work: Opportunities and barriers. Am J Med. 2014;127(3):183-7. https://doi. org/10.1016/j.amjmed.2013.09.032

37. Rudowitz R. COVID-19: Expected implications for Medicaid and state budgets. Kaiser Family Foundation. Available from: https://www.kff.org/coronavirus-policywatch/covid-19-expected-implicationsmedicaid-state-budgets/

Copyright Ownership: This is an open access article distributed in accordance with the Creative Commons Attribution Non Commercial (CC BY-NC 4.0) license, which permits others to distribute, adapt, enhance this work non-commercially, and license their derivative works on different terms, provided the original work is properly cited and the use is noncommercial. See: http://creativecommons. org/licenses/by-nc/4.0. 